\title{
First Long-Term Application of Squeezed States of Light in a Gravitational-Wave Observatory
}

\author{
H. Grote,, , K. Danzmann, ${ }^{1}$ K.L. Dooley, ${ }^{1}$ R. Schnabel, ${ }^{1}$ J. Slutsky, ${ }^{1}$ and H. Vahlbruch ${ }^{1}$ \\ ${ }^{1}$ Max-Planck-Institut für Gravitationsphysik (Albert Einstein Institut) und \\ Leibniz Universität Hannover, Callinstr. 38, $3016^{7}$ Hannover, Germany
}

(Dated: August 14, 2018)

\begin{abstract}
We report on the first long-term application of squeezed vacuum states of light to improve the shot-noise-limited sensitivity of a gravitational-wave observatory. In particular, squeezed vacuum was applied to the German / British detector GEO 600 during a period of three months from June to August 2011, when GEO 600 was performing an observational run together with the French / Italian Virgo detector. In a second period squeezing application continued for about 11 months from November 2011 to October 2012. During this time, squeezed vacuum was applied for 90.2\% (205.2 days total) of the time that science-quality data was acquired with GEO 600. Sensitivity increase from squeezed vacuum application was observed broad-band above $400 \mathrm{~Hz}$. The time average of gain in sensitivity was $26 \%(2.0 \mathrm{~dB})$, determined in the frequency band from $3.7 \mathrm{kHz}$ to $4.0 \mathrm{kHz}$. This corresponds to a factor of two increase in observed volume of the universe, for sources in the $\mathrm{kHz}$ region (e.g. supernovae, magnetars). We introduce three new techniques to enable stable long-term application of squeezed light, and show that the glitch-rate of the detector did not increase from squeezing application. Squeezed vacuum states of light have arrived as a permanent application, capable of increasing the astrophysical reach of gravitational-wave detectors.
\end{abstract}

PACS numbers: 04.80.Nn, 95.55.Ym, 95.75.Kk, 42.50.Lc

Gravitational waves were predicted by Albert Einstein as a consequence of his General Theory of Relativity [1], but have not been directly measured to date. While a number of large-scale laser-interferometers such as LIGO [2], Virgo [3], TAMA [4], and GEO 600 [5], were (or are, in the case of GEO 600) in operation, their sensitivities were not yet sufficient to make the first direct detection of gravitational waves $(\mathrm{GW})$. One of the sensitivity limits of these instruments originates from the zeropoint fluctuation of the quantum vacuum state of light. While this noise can in principle be reduced with respect to the signal size by the use of higher laser power, this method is ultimately limited by thermal deformation of optical components and other laser power related instabilities [6, 7]. It was proposed by Caves [8] that quantum noise in the shot-noise regime can also be reduced with the injection of squeezed vacuum states into the otherwise open output port of an interferometer. It took several years until squeezed-light sources became available and to demonstrate this principle on table-top interferometers [9-12]. Even more time was required to develop squeezed-light sources that made squeezed states at audio frequencies available, which is the frequency band relevant for the $\mathrm{km}$-scale interferometers [13 16]. Squeezing above the audio band was demonstrated in a prototype GW-interferometer [17] with suspended mirrors, and the first demonstration of quantum-enhancement in one of the gravitational-wave observatories was done in 2010 in the GEO 600 detector [18]. Squeezing of the LIGO observatory in Hanford, WA, was then demonstrated in

*Electronic address: hartmut.grote@aei.mpg.de late 2011 [19]. While all of these experiments were onetime demonstrations of squeezed vacuum applied on time scales of minutes, in this work we report on the first continuous (and ongoing) application of squeezed light to a gravitational-wave interferometer, namely GEO 600, to enhance the shot-noise limited sensitivity.

The GEO detector is currently the only operational laser-interferometric GW detector worldwide and is acquiring calibrated measurement data in the so-called astrowatch mode. Data taken in this mode covers about $2 / 3$ of the total time, when regular work as part of an incremental upgrade program called GEO-HF [20] does not take place. The search for gravitational-wave signals in the astrowatch data will be triggered only by the occurrence of external (e.g. electromagnetic or neutrino) signals, as expected e.g. from a supernova in our galaxy.

GEO 600 [5, 21] is a Michelson interferometer with folded $600 \mathrm{~m}$ long arms, using the advanced technique of dual recycling [22] (power- and signal recycling). Figure 1 shows a simplified layout of GEO 600, together with the squeezed-light source [23, 24]. The squeezed states are injected into the main interferometer via a Faraday Isolator and must be mode-matched and aligned to the main interferometer beam. In addition, two servo systems (denoted in Fig. 1 as 'Phase locked loop' and 'Squeezing phase feedback') are necessary to assure the squeezing is at the correct optical laser frequency and detected interferometer output quadrature. Through the implementation of three new techniques for alignment and squeezing phase control, we achieve a stable and long-term application of squeezed light to a GW observatory. Figure 2 shows typical strain spectral densities of GEO 600, comparing the non-squeezed noise floor with the squeezed noise floor, at the end of August 2012. Shot-noise reduc- 


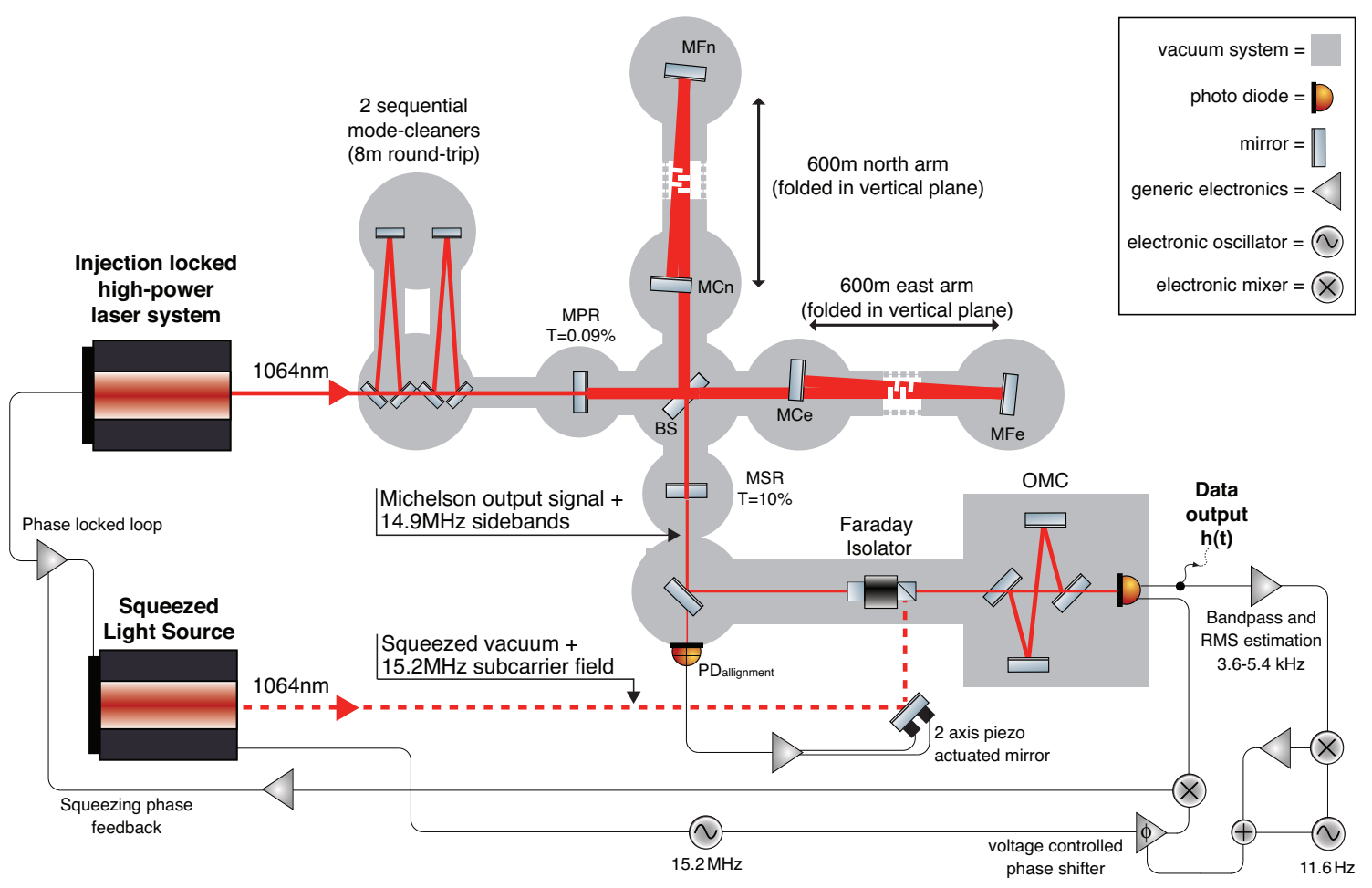

FIG. 1: A simplified optical layout of the squeezed-light enhanced gravitational wave detector GEO 600, which consists of the conventional GEO 600 observatory and the additional squeezed-light source. The observatory has two singly folded arms with a total optical length of $2400 \mathrm{~m}$. A gravitational wave passing from most directions will shorten one arm, while the length of the perpendicularly orientated arm is increased, and vice versa in the next half-cycle of a passing wave, producing a periodic power change of the output light that is detected by a photo diode. The observatory is operated such that almost of all the light is back-reflected towards the input laser by keeping the interferometer output close to a dark fringe via a control system. A power-recycling mirror (MPR) leads to a resonant enhancement of the circulating light power of $2.7 \mathrm{~kW}$ at the beam splitter. Similar to the power-recycling technique, a partially transmissive signal-recycling mirror (MSR) is installed to further resonantly enhance the GW-induced signal at the interferometer's output. BS: 50/50 beam splitter, MFe/MFn: far interferometer end mirrors (east/north), MCe/MCn: central interferometer mirrors, T: mirror power transmissivity. All interferometer optics are situated in a vacuum system and suspended by multi-stage pendulums.

tion is observed at frequencies higher than about $400 \mathrm{~Hz}$.

We apply an automatic alignment system for a squeezed vacuum field for the first time. Because all of the GEO 600 core optics are suspended as pendula to reduce seismic perturbations, the alignment of the squeezed field into the interferometer must continuously follow the spatially drifting GEO 600 output field. An error signal for this control loop is derived by sensing the optical beat between the $15.2 \mathrm{MHz}$ frequency shifted squeezed vacuum control field [14] and the $14.9 \mathrm{MHz}$ Michelson interferometer sidebands leaving the interferometer's dark port 21], as illustrated in Fig. 1. The servo feedback signals are sent to a 2 -axis piezo actuated mirror, placed in the squeezing injection path, suppressing relative motions up to a frequency of $3 \mathrm{~Hz}$.

Furthermore, we introduce a new control signal for the phase relation between the injected squeezed field and the interferometer output field. We derive an error signal using the detected light transmitted through the output mode-cleaner (OMC) where the squeezing coherent control sidebands [14] beat with the spatially filtered
GEO 600 carrier light (TEM00-mode). The reduction of higher order modes in the filtered GEO output beam, as well as the fact that all of the output TEM00-mode carrier light is available to use, results in a superior error signal to shot noise ratio, as compared to previously used techniques. Although the detected squeezing level is currently primarily limited by optical loss [18], employing this new control signal already improves the strain sensitivity of GEO 600. As optical losses are reduced, which is foreseen during the GEO-HF upgrade, phase noise will play a more significant role in limiting the detected squeezing level, thus making the benefits of this technique more important. The new control signal will also be of particular interest to the third generation laser interferometeric GW-detectors 25] where the goal is to detect up to $10 \mathrm{~dB}$ of squeezing.

Finally, the coherent control scheme for controlling the squeezer phase is extended to include a noise locking scheme [26] to compensate for slow drifts of the optimal squeezing angle. The implementation of this noise lock is similar to the one described in [26], but the com- 


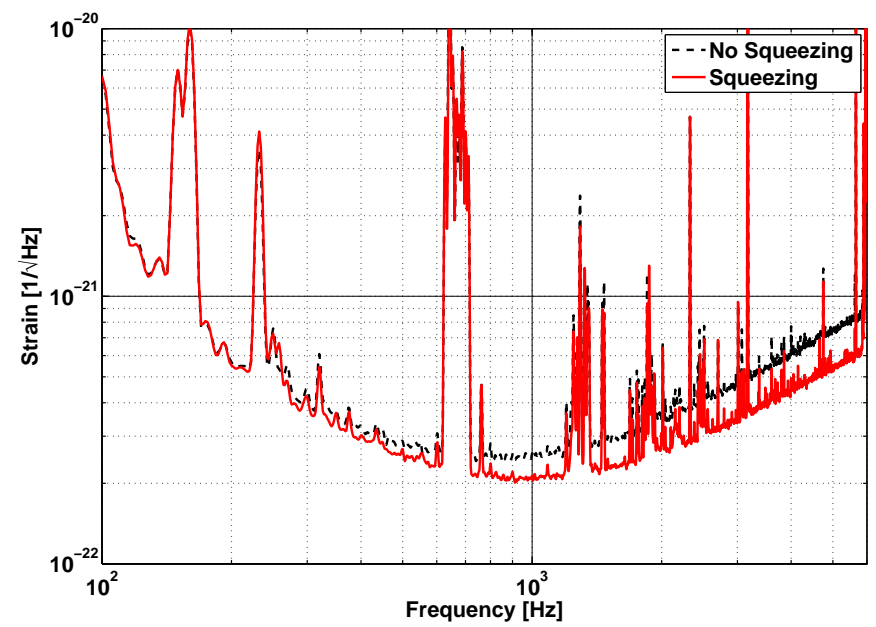

FIG. 2: Strain amplitude spectral densities of GEO 600 with and without squeezing being applied. This measurement reflects the state of squeezing application at the end of August 2012 , as an example for the squeezing performance during the time from November 2011 to October 2012. The large structures from $620-720 \mathrm{~Hz}$ are unresolved lines caused by vibrational modes of the wires suspending the test masses.

bination with the coherent control scheme is used for the first time. The demodulation phase of the squeezing phase control signal is electronically modulated at a frequency of $11.6 \mathrm{~Hz}$. In order to maximize the detected squeezing level, as measured in a frequency band between $3.6 \mathrm{kHz}$ and $5.4 \mathrm{kHz}$ of the $\mathrm{h}(\mathrm{t})$ data stream, a control loop with unity gain at about $10 \mathrm{mHz}$ corrects for drifts of the optimal demodulation phase over long timescales. The unity gain frequency of the coherent control loop is about $1 \mathrm{kHz}$.

The three new techniques described above were implemented in stages during two long-term GEO 600 science data taking periods. From June 3 to September 5, 2011, GEO 600 took part in an observational run, named S6e/VSR4, together with the Virgo detector. During the 93.3 days of this run, no detector commissioning work was performed. As a result, science-quality data were acquired for $84.3 \%$ (78.8 days) of the total time, with squeezing applied for $53.1 \%$ (41.8 days) of the science time. The average squeezing level observed in this period was $16 \%(1.3 \mathrm{~dB})$.

In the second period lasting from November 17, 2011 to October 15, 2012, the GEO 600 observatory took data in regular astrowatch mode. Compared to the S6e/VSR4 run, we achieved a much higher squeezing application time, as well as an improved observed average squeezing level. The astrowatch time obtained in this period of 333 days was $68.2 \%$ (227.4 days) with squeezed vacuum being applied for $90.2 \%$ (205.2 days) of the astrowatch time with an average gain in detector sensitivity of $26 \%$ $(2.0 \mathrm{~dB})$. For the last two weeks of this period we achieved the highest duty factor so far: squeezing was applied to $99.66 \%$ of the astrowatch science-quality data, demon-

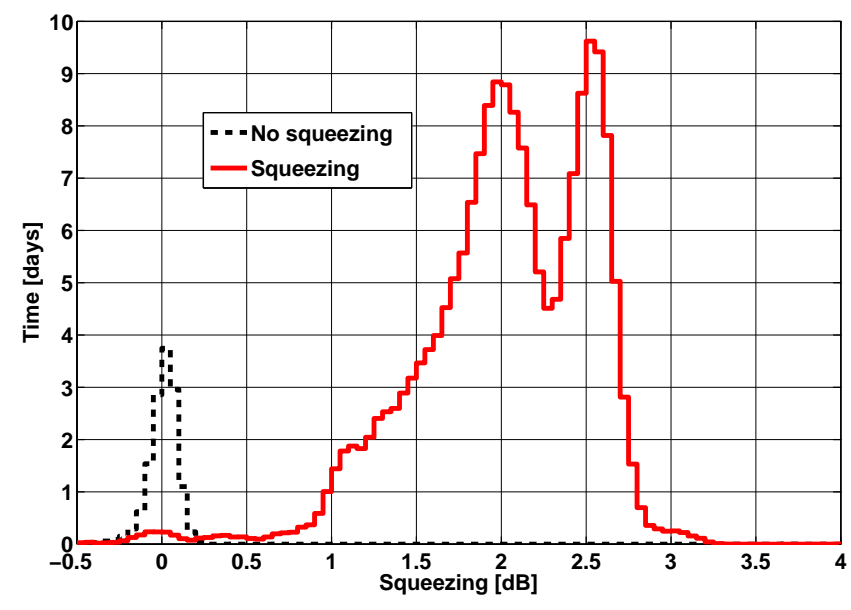

FIG. 3: Histogram of the observed squeezing at GEO 600 in the band from 3692 to $3957 \mathrm{~Hz}$. A median of $26 \%(2.0 \mathrm{~dB})$ of squeezing was achieved when squeezing was applied. This histogram contains the full data set from November 17, 2011 to October 15, 2012. The different squeezing levels are caused mainly by different operating conditions of the squeezing application. The larger squeezing level of about $2.5 \mathrm{~dB}$ is mainly from the latest time in the period reported here when all new control techniques were being used.

strating the success of the new control techniques.

In order to characterise the effect of the squeezing application we look at the frequency dependent increase in sensitivity, as well as the stationarity of the data. We use the Omega data analysis pipeline 27] for these investigations, which calculates the amplitude spectral density of 64-second data intervals, overlapping by 8 seconds, starting from the beginning of each science data segment. For the squeezing level estimation we compare this amplitude spectrum with the expected un-squeezed shot-noise amplitude spectrum, evaluating for the frequency range from $3692 \mathrm{~Hz}$ to $3957 \mathrm{~Hz}$. This range is chosen as it is clean of spectral lines and other noise contributions, simplifying the long term calculation of the squeezing. However, the suppression is broadly the same in the $2 \mathrm{kHz}$ to $6 \mathrm{kHz}$ regime (see also Figure 21). Figure 3 shows the result for the period from November 17, 2011 to October 15,2012 . The average gain in sensitivity from squeezed vacuum application in this period was $26 \%(2.0 \mathrm{~dB})$. This improvement in sensitivity corresponds to an increase of the observable volume of the universe by a factor of two for gravitational-wave sources in this frequency band.

To investigate the noise behavior on short time scales (i.e. 1 second and below) the Omega pipeline uses the $64 \mathrm{~s}$ long spectra to whiten the data, and then performs a wavelet analysis to search for transients, which are thresholded and clustered into triggers. These transients, named glitches for our purpose, degrade our ability to detect potential gravitational-wave signals with similar time-frequency parameters. Glitches occur in all gravitational-wave detectors and can have many differ- 


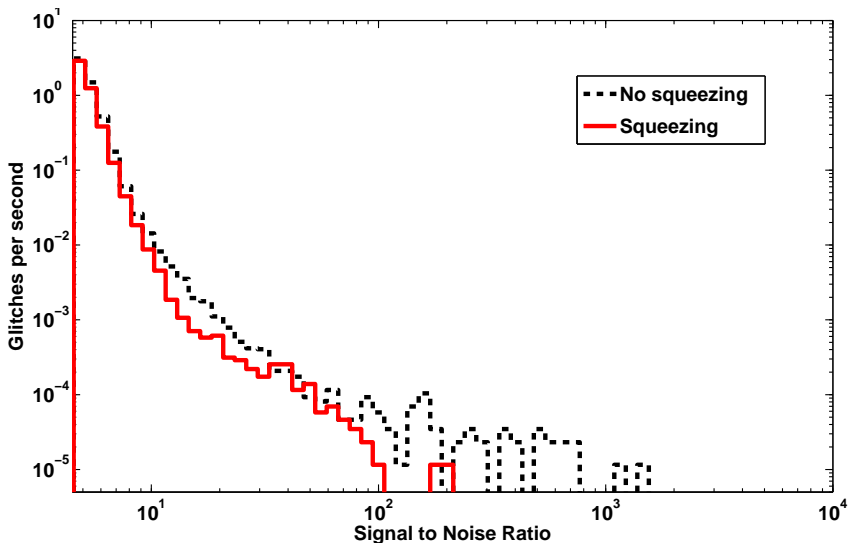

FIG. 4: Histograms of the signal-to-noise ratio (SNR) of glitches in the gravitational-wave channel. Two data sets of 24 hours duration each are compared for the cases of squeezing being applied and not being applied, respectively. No increase in glitch rate can be observed for the case of squeezing being applied. The reduction in glitch rate when squeezing was applied is not considered significant with respect to the usage of squeezing.

ent origins (i.e. optical, electrical, etc., see e.g. [28]). Any new technique added to a gravitational-wave detector should not increase the glitch rate, which would compromise the potential utility of the technology.

Figure 4 shows histograms of signal-to-noise ratios for glitches detected during two 24 hour reference periods, one each for squeezing and no squeezing applied, constituting the first investigation of this kind. Neither the rate nor outlier amplitude of the glitches was observed to increase for the interval with squeezing applied, compared with the time without the application of squeezing. This data without the application of squeezing was created intentionally to examine the effect of the squeezer on the glitch population in the detector data. No other deliberate attempt was made to set aside data without squeezing, but investigation of a similar data stretch from months earlier when squeezing was unavailable for technical reasons yielded consistent results.

We will continue to apply squeezed vacuum as much as possible during the next years, while also trying to increase the level of observed squeezing. Currently, the main limitation in the level of observed squeezing comes from optical losses in the output-mode cleaner.

We conclude that we have demonstrated the application of squeezed vacuum states over a period of more than one year, enhancing the shot-noise limited sensitivity of a laser-interferometric gravitational-wave observatory. Squeezed vacuum states are now regularly used at GEO 600.

\section{Acknowledgments}

We thank the GEO collaboration for the construction of GEO 600 and the quantum interferometry group for the development of the squeezed light source and its technology. The authors are also grateful for support from the Science and Tech. Facilities Council (STFC), the Univ. of Glasgow in the UK, the Max Planck Society, the Bundesm. f. Bildung und Forschung (BMBF), the Volkswagen Stiftung, the cluster of excellence QUEST, the Max Planck Research School (IMPRS), the State of Nds. in Germany, and DFG grant SFB/TR 7.
[1] Einstein,A. Annalen der Physik 49, 769-822 (1916).

[2] Abbott,B. P. et al. Rep. Prog. Phys. 72, 076901 (2009).

[3] Accadia,T. et al. Journal of Instrumentation 7, P03012 (2012).

[4] Arai,K. et al. Class. Quantum Grav. 26, 204020 (2009).

[5] Willke,B. et al. Class. Quantum Grav. 19, 1377-1387 (2002).

[6] DAmbrosio,E. Phys. Rev. D 67, 102004 (2003).

[7] Zhao,C. Ju,L. Degallaix,J. Gras,S. Blair,D.G. Phys. Rev. Lett. 94121102 (2005)

[8] Caves,C. M. Phys. Rev. D 23, 1693-1708 (1981).

[9] Xiao,M. Wu,L.A. \& Kimble, H. J. Phys. Rev. Lett. 59, 278-281 (1987).

[10] Grangier,P. Slusher,R.E., Yurke,B. \& LaPorta,A. Phys. Rev. Lett. 59, 2153-2156 (1987).

[11] McKenzie,K. Shaddock,D. A. McClelland,D. E. Buchler, B. C. \& Lam,P. K. Phys. Rev. Lett. 88, 231102 (2002).

[12] Vahlbruch,H. Chelkowski,S. Hage,B. Franzen,A. Danzmann,K. \& Schnabel,R. Phys. Rev. Lett. 95, 211102 (2005).

[13] McKenzie,K. et al. Phys. Rev. Lett. 93, 161105 (2004).

[14] Vahlbruch,H. Chelkowski,S. Hage, B. Franzen,A. Danzmann,K. \& Schnabel,R. Phys. Rev. Lett. 97, 011101 (2006).
[15] Vahlbruch,H. Chelkowski,S. Danzmann,K. Schnabel,R. New J. Phys. 9, 371 (2007).

[16] Vahlbruch,H. et al. Phys. Rev. Lett. 100, 033602 (2008).

[17] Goda,K. et al. Nature Physics 4, 472-476 (2008).

[18] The LIGO Scientific Collaboration, Nature Physics 7, 962-965 (2011)

[19] The LIGO Scientific Collaboration to be published

[20] Lück, H. et al. J. Phys. Conf. Ser. 228, 012012 (2010).

[21] Grote,H. et al. Class. Quantum Grav. 27, 084003 (2010).

[22] Meers,B.J. Phys. Rev. D 38, 2317-2326 (1988).

[23] Vahlbruch,H. Khalaidovski,A. Lastzka,N. Gräf,C. Danzmann,K. \& Schnabel,R. Class. Quantum Grav. 27, 084027 (2010).

[24] Khalaidovski,A. Vahlbruch,H. Lastzka,N. Gräf,C. Danzmann,K. Grote,H. \& Schnabel,R. Class. Quantum Grav. 29, 075001 (2012).

[25] Hild, S. et al. Class. Quantum Grav. 28, 094013 (2011).

[26] McKenzie, K. et al. J. Opt. B: Quant. Semiclass. Opt. 7, 421-428 (2005).

[27] Chatterji,S. Blackburn,L. Martin,G. and Katsavounidis,E. Class. Quant. Grav. 21 1809-1818 (2004)

[28] Aasi,J. et al. Class. Quantum Grav. 29155002 (2012) 